\title{
Murine Double Minute-2 Inhibition Ameliorates Established Crescentic Glomerulonephritis
}

Shrikant R. Mulay, Simone Romoli, Jyaysi Desai, Mohammad Mohsen Honarpisheh, Santhosh V. Kumar, Hans-Joachim Anders, and Dana Thomasova

From the Division of Nephrology, Medizinische Klinik und Poliklinik IV, University Hospital of Ludwig-Maximilians-University, Munich, Germany

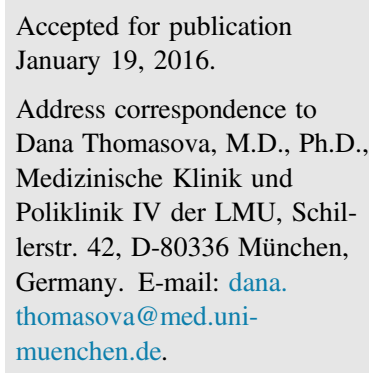

\begin{abstract}
Rapidly progressive glomerulonephritis is characterized by glomerular necroinflammation and crescent formation. Its treatment includes unspecific and toxic agents; therefore, the identification of novel therapeutic targets is required. The E3-ubiquitin ligase murine double minute (MDM)-2 is a nonredundant element of NF- $\kappa B$ signaling and the negative regulator of tumor suppressor gene TP53-mediated cell cycle arrest and cell death. We hypothesized that the MDM2 would drive crescentic glomerulonephritis by $\mathrm{NF}-\kappa \mathrm{B}$-dependent glomerular inflammation and by $\mathrm{p53}$-dependent parietal epithelial cell hyperproliferation. Indeed, the pre-emptive MDM2 blockade by nutlin-3a ameliorated all aspects of crescentic glomerulonephritis. MDM2 inhibition had identical protective effects in Trp53-deficient mice, with the exception of crescent formation, which was not influenced by nutlin-3a treatment. In vitro experiments confirmed the contribution of MDM2 for induction of NF- $\kappa \mathrm{B}-$ dependent cytokines in murine glomerular endothelial cells and for p53-dependent parietal epithelial cell proliferation. To evaluate MDM2 blockade as a potential therapeutic intervention in rapidly progressive glomerulonephritis, we treated mice with established glomerulonephritis with nutlin-3a. Delayed onset of nutlin-3a treatment was equally protective as the pre-emptive treatment in abrogating crescentic glomerulonephritis. Together, the pathogenic effects of MDM2 are twofold, that is, p53-independent NF- $\kappa$ B activation increasing intraglomerular inflammation and p53-dependent parietal epithelial cell hyperplasia and crescent formation. We therefore propose MDM2 blockade as a potential novel therapeutic strategy in rapidly progressive glomerulonephritis. (Am J Pathol 2016, 186: 1442-1453; http://dx.doi.org/10.1016/j.ajpath.2016.01.017)
\end{abstract}

Rapidly progressive glomerulonephritis (GN) is clinically defined by rapid loss of glomerular filtration rate together with proteinuria and hematuria as biomarkers of glomerular filtration barrier disruption. ${ }^{1}$ This clinical syndrome can be a manifestation of ANCA-associated vasculitis, anti-glomerular basement membrane (GBM) disease, or immune complex $\mathrm{GN},{ }^{2}$ which all trigger glomerular vascular necrosis by neutrophil extracellular trap formation and the local cytotoxicity of extracellular histones and complement. ${ }^{3,4}$ Glomerular loop necrosis results in plasma leakage into Bowman space, causing hyperplasia of parietal epithelial cells (PECs), that is, glomerular crescent formation. ${ }^{5}$ Current treatments of rapidly progressive GN include unspecific, and therefore, toxic immunosuppressant and antiproliferative agents such as steroids and cyclophosphamide. It remains an unmet medical need to identify more specific molecular targets that mediate both glomerular necroinflammation and PEC hyperproliferation in rapidly progressive $\mathrm{GN}^{6}$

The E3 ubiquitin ligase murine double minute-2 (MDM2) regulates proteasomal degradation of the tumor suppressor gene and cell cycle regulator $\mathrm{p} 53$ but also facilitates NF- $\kappa \mathrm{B}$ signaling. ${ }^{7,8}$ MDM2 promotes the survival and proliferation of malignant cells by p53 inhibition. ${ }^{9-11}$ Vice versa, MDM2 blockade promotes cell cycle arrest or cell death by enhanced p53 activation. To use these properties, specific small molecule MDM2 inhibitor nutlins were developed and are currently in clinical trials for cancer treatment. ${ }^{11}$ We have

Supported by Deutsche Forschungsgemeinschaft grants TH1836/1-2 (D.T.) and AN372/9-2 (H.-J.A.).

S.R.M. and S.R. contributed equally to this work.

H.-J.A. and D.T. contributed equally to this work as senior authors.

Disclosures: None declared. 
previously reported that MDM2 drives NF- $\kappa \mathrm{B}$ signalingrelated sterile inflammation in acute tubular injury, whereas its proregenerative effect in the healing phase was exclusively p53 dependent. ${ }^{7}$ In podocyte injury, MDM2 promotes glomerular inflammation and podocyte loss by overcoming cell cycle $\mathrm{G}_{2} / \mathrm{M}$ arrest and entering mitosis (ie, mitotic catastrophe). ${ }^{12}$ The anti-inflammatory and antiproliferative potential of MDM2 inhibition can elicit additive effects, in particular in lymphoproliferative autoimmune diseases, such as systemic lupus erythematosus and lupus nephritis. ${ }^{13}$

We therefore hypothesized that MDM2 would drive crescentic GN by NF- $\kappa \mathrm{B}$-dependent glomerular inflammation and by $\mathrm{p} 53$-dependent PEC hyperproliferation and that, vice versa, MDM2 blockade could be a potent and innovative strategy to abrogate crescentic GN.

\section{Materials and Methods}

\section{Animals and Experimental Design}

C57BL6/N mice were procured from Charles River Laboratories (Sulzfeld, Germany), and p53-deficient C57BL6/N mice were obtained from Taconic (Ry, Denmark). Mice were housed in groups of five in standard housing condition with a 12-hour light/dark cycle. Cages, nest lets, food, and water were sterilized by autoclaving before use, and mice were allowed unlimited access to food and water. Six- to 8-weekold mice received an intravenous injection of $75 \mu \mathrm{L}$ of antiGBM serum (PTX-001 sheep anti-rat GBM serum; Probetex, Inc., San Antonio, TX). Mice received intraperitoneal injections with $20 \mathrm{mg} / \mathrm{kg}$ of the MDM2 inhibitor nutlin-3a (Enzo Life Sciences, Lörrach, Germany) in 50\% dimethyl sulfoxide (vehicle) or vehicle only, every alternate day. On day 7 , mice were euthanized by cervical dislocation, and kidney tissues were harvested. Blood and urine were collected before sacrifice to evaluate the functional markers of the kidney damage. A part of the kidneys was stored at $-80^{\circ} \mathrm{C}$ for protein isolation and later in RNA solution at $-20^{\circ} \mathrm{C}$ for RNA isolation. A part of the kidney was also fixed in formalin to be embedded in paraffin for histologic analysis. The G*Power software version 3 (Heinrich Heine University, Dusseldorf, Germany) was used to calculate sample size for the experiments performed. An effect size of $0.9, \alpha$ error of 0.05 , and power of 0.8 was considered for these calculations. Mice were assigned different groups with the use of stratified randomization method. ${ }^{14}$ Humane end points, as in the ethical approval for the study, were monitored throughout the study. All animal studies were conducted according to the European equivalent of the NIH's Guide for the Care and Use of Laboratory Animals and were approved by the local governmental authorities. ${ }^{15}$ Urine albumin concentrations (Bethyl Laboratories, Montgomery, TX), urine and plasma creatinine concentrations and blood urea nitrogen (BUN) (DiaSys GmBH, Holzheim, Germany) concentrations were determined with commercially available kits according to the manufacturer's protocol.
Renal Structure, Immunohistochemistry, and Confocal Microscopy

Kidney tissues were fixed in $4 \%$ neutral-buffered formalin, dehydrated in graded alcohols, and embedded in paraffin. For routine histologic and morphometric analyses $4-\mu \mathrm{m}$ sections were stained with periodic acid-Schiff reagent. For immunohistochemistry sections were deparaffinized, rehydrated, transferred into citrate buffer, and either autoclaved or microwave-treated for antigen retrieval and processed as described. $^{7}$ The following primary antibodies were used: anti-mouse Ly6G, anti-mouse F4/80 (both Serotec, Oxford, UK), anti-mouse mac-2 (Cedarlane Laboratories, Toronto, ON, Canada), pig anti-mouse nephrin (dilution 1:100; Acris Antibodies, Herford, Germany), rabbit anti-mouse WT1 (dilution 1:25; Santa Cruz Biotechnology, Santa Cruz, CA), rabbit anti-mouse MDM2, anti-mouse fibrinogen (dilution 1:100; Abcam, Cambridge, UK), rat anti-mouse CD31 (dilution 1:100; Dianova, Hamburg, Germany), and rabbit anti-mouse claudin-1 (dilution 1:100; Bioworld Technologies, St. Louis Park, MN). Immunofluorescence staining was evaluated with LSM 510 confocal microscope and LSM software version 4.2 (Carl Zeiss, Jena, Germany). Nuclei were counterstained with To-pro-3 (Life Technologies, Carlsbad, CA). The degree of glomerulosclerosis was assessed by observing 50 randomly selected glomeruli from each mouse kidney from periodic acid-Schiff-stained kidney sections under a light microscope (Leitz DMR; Leica Microsystems, Bensheim, Germany). These glomeruli were then further classified as having no lesions, segmental lesions $(<50$ of glomerulus), or global lesions ( $>50 \%$ of glomerulus). Cellular crescents were assessed separately, when more than a single layer of PECs was present around the inner circumference of Bowman capsule. Immunostaining positivity was quantified with Photoshop software version CS6 (Adobe Systems, San Jose, CA) and expressed as percentage of total area.

\section{RNA Preparation and Real-Time Quantitative PCR}

Total RNA was isolated from kidneys with the use of Ambion RNA extraction kit (Invitrogen, Carlsbad, CA) according to the manufacturer's instructions. After quantification, RNA quality was assessed with agarose gels before reverse transcription with Superscript II (Invitrogen). Real-time RT-PCR was performed with SYBRGreen PCR master mix and was analyzed with a Light Cycler 480 (Roche, Mannheim, Germany). All gene expression values were normalized with 18s RNA as a house keeping gene. All primers used for amplification were from Metabion (Martinsried, Germany). Primer sequences used are listed in Table 1.

\section{Protein Isolation and Western Blot Analysis}

We extracted protein from kidney tissues with the use of RIPA buffer (Sigma-Aldrich, Hamburg, Germany) that contained protease inhibitors (Roche) and processed it for Western blot 
Table 1 Primers Used for Real-Time PCR

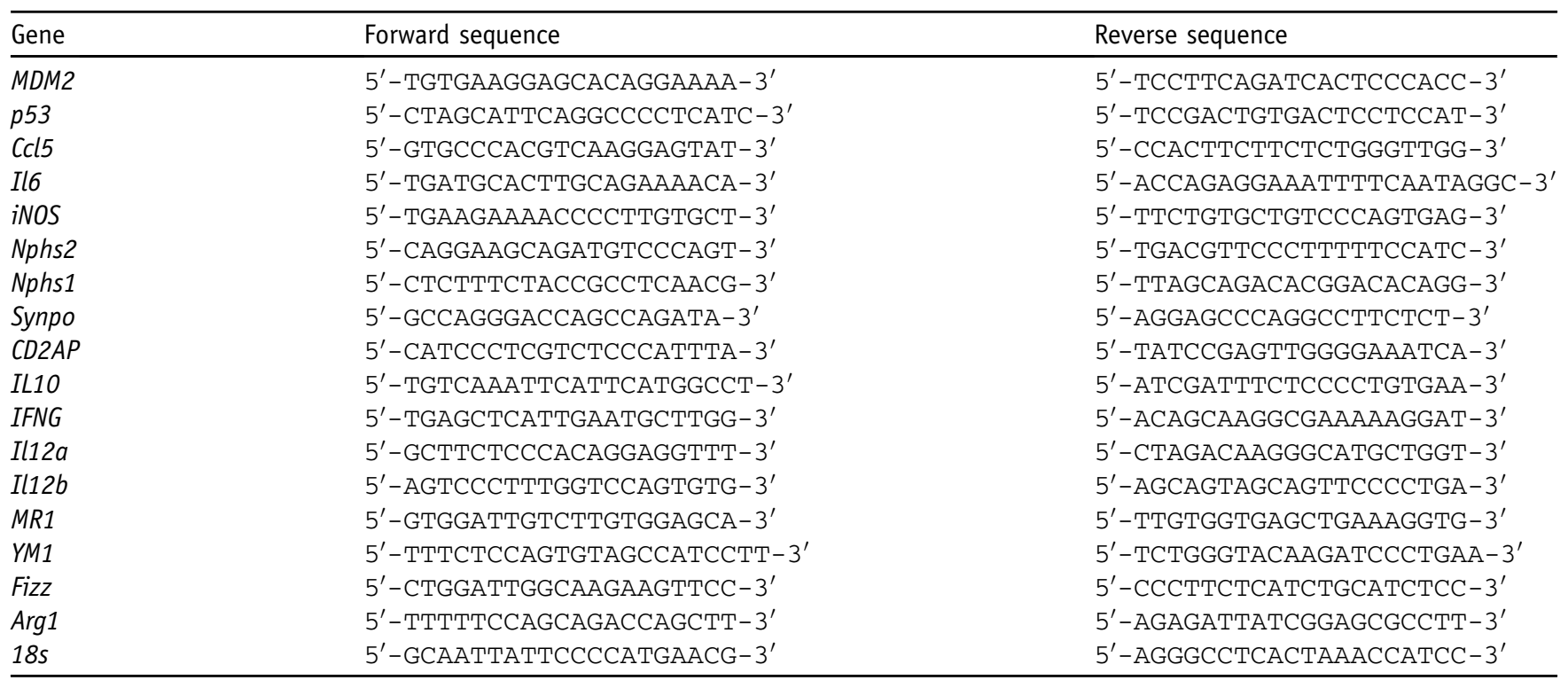

analysis as described. ${ }^{7}$ The membranes were incubated overnight at $4^{\circ} \mathrm{C}$ with primary antibodies, goat antibody against mouse p53 (Santa Cruz Biotechnology), rabbit antibody against MDM-2 (Abcam), phosphorylated-53 (Santa Cruz Biotechnology), phosphorylated-MDM2 (Cell Signaling Technology Inc., Danvers, MA), and rabbit antibody against $\beta$-actin (Cell Signaling Technology Inc.). Secondary antibodies were a peroxidase-conjugated donkey anti-goat IgG (Dianova) and an anti-rabbit IgG (Cell Signaling Technology Inc.).

\section{Cell Culture Studies}

Mouse PECs and murine glomerular endothelial cells (GEnCs) were cultivated as described elsewhere. ${ }^{16,17}$ GEnCs for RNA extraction were seeded at a density of $5 \times 10^{5}$ cells/well in 6-well plates in DMEM medium and grown overnight to confluence. The cells were then incubated with $25 \mathrm{nmol} / \mathrm{L}$ MDM2 or control siRNA for 24 hours with the use of Lipofectamine RNAiMAX transfection reagent (Life Technologies, Carlsbad, CA) for transient transfection. Half of the transfected cells were treated with $5 \mu \mathrm{g} / \mathrm{mL}$ ultrapure lipopolysaccharide (LPS; InvivoGen, San Diego, CA), incubated at $37^{\circ} \mathrm{C}, 5 \% \mathrm{CO}_{2}$ for 18 hours. Then both the stimulated and unstimulated transfected GEnCs were harvested for mRNA preparation and real-time quantitative PCR analysis of proinflammatory genes. For the proliferation/viability assays, the mouse PECs were seeded in 96-well plates (5000 cells/well) and kept under nonpermissive conditions $\left(37^{\circ} \mathrm{C}\right.$ in growth medium without interferon- $\gamma$ ) for 12 days to induce the differentiation of PECs. The PECs were then incubated with $25 \mathrm{nmol} / \mathrm{L}$ p53 or control siRNA for 24 hours for transient transfection and then treated with nutlin-3a 10,20 , or $30 \mu \mathrm{mol} / \mathrm{L}$ or dimethyl sulfoxide for 24 hours, and MTT assay was performed (CellTiter 96 Non-Radioactive Cell Proliferation Assay; Promega, Madison, WI) according to the manufacturer's instructions. Experiments were performed in triplicate. Specific siRNA to silence selectively murine or murine p53 and appropriate control siRNA (negative control no. 1) were purchased from Ambion (Life Technologies).

\section{Luciferase Reporter Assay}

The PECs and GEnCs were transfected with NF- $\kappa \mathrm{B}-\mathrm{luc}$ and Renilla reporter plasmids with the use of nucleofection device. Twenty-four hours after transfection the cells were pretreated with nutlin-3a for 1 hour before inducing NF- $\kappa \mathrm{B}$ signaling with $1 \mu \mathrm{g} / \mathrm{mL}$ LPS or vehicle for 5 hours. The cells were then processed with the use of the dual luciferase reporter system according to the manufacturer's protocol (Promega). The GEnCs were additionally treated with $30 \mu \mathrm{mol} / \mathrm{L}$ pifithrin- $\alpha$, p53 chemical inhibitor. Activities of firefly and Renilla luciferases were measured by luminometer. Firefly luciferase activity was divided by Renilla luciferase activity to correct for differences in transfection efficiencies.

\section{Statistical Analysis}

Values are expressed as means \pm SD. Statistical analysis was performed with GraphPad Prism software version 5 (GraphPad Inc., San Diego, CA). Significance of differences was determined by the appropriate two-sided $t$-test for single comparisons. Analysis of variance with post hoc Bonferroni correction was used for multiple comparisons. $P<0.05$ was considered statistically significant.

\section{Results}

\section{MDM2 Expression in Crescentic GN}

First, we characterized the expression of MDM2 and p53 in crescentic GN 7 days after sheep GBM antiserum 
A

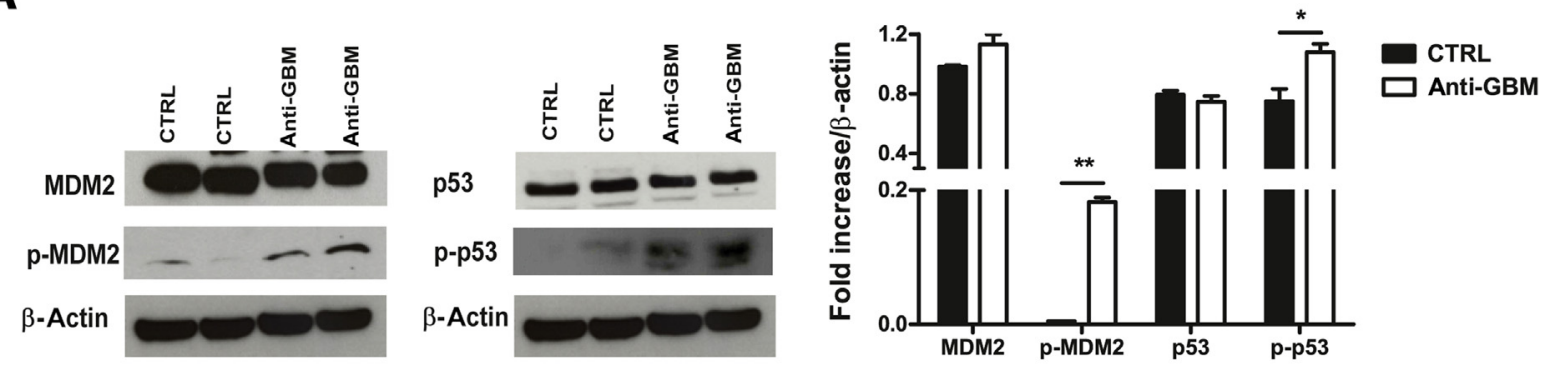

B
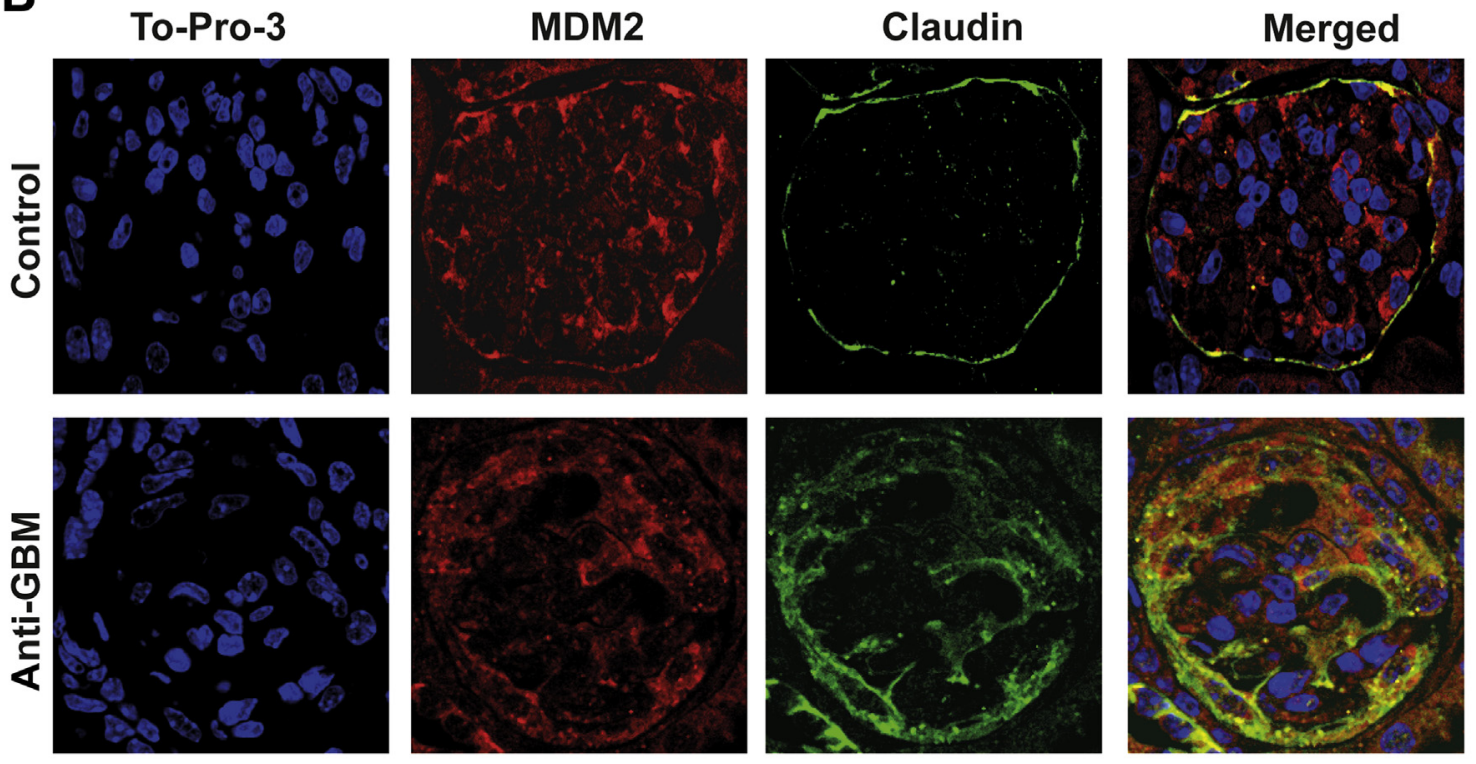

\section{C}
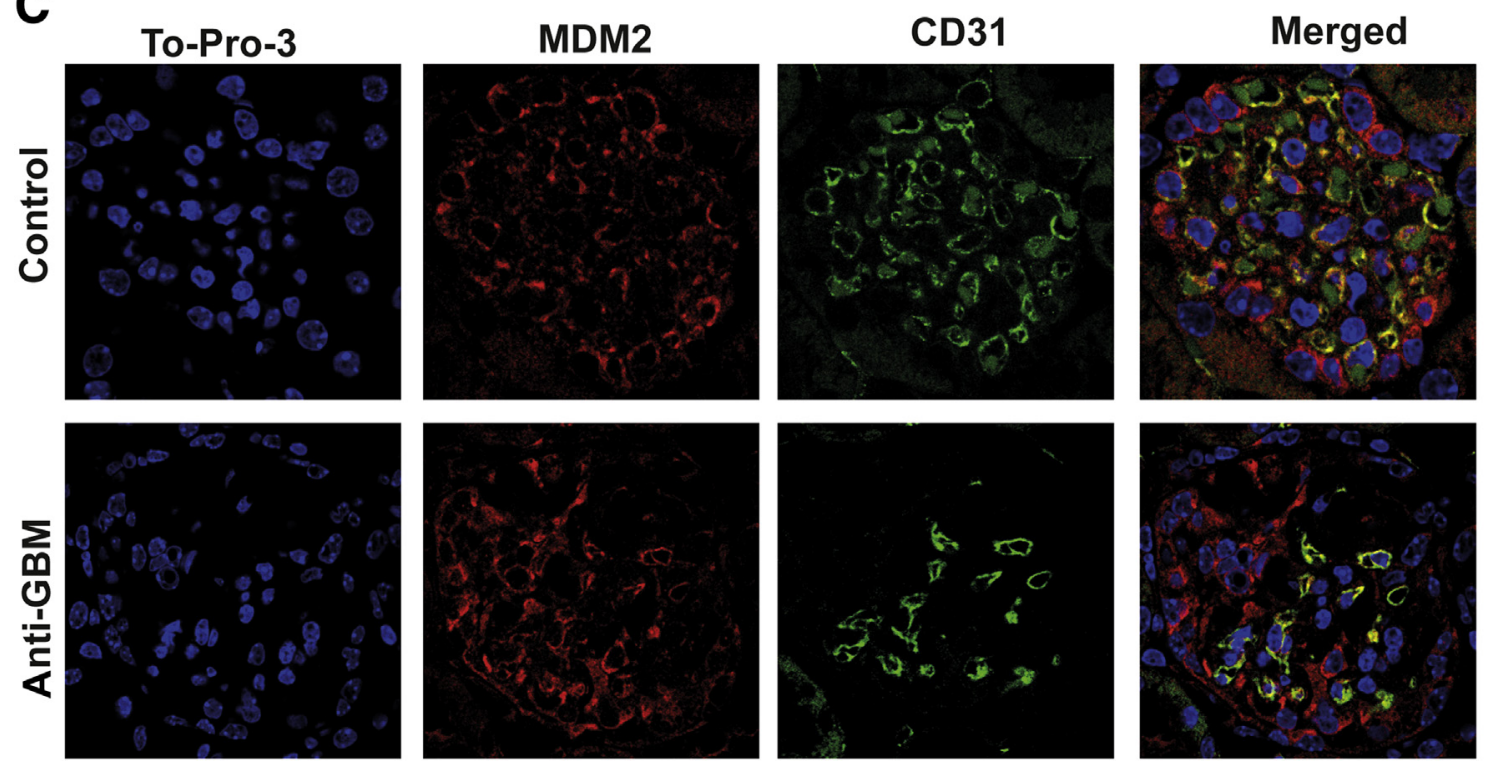

Figure 1 Renal MDM2 expression. A: The expression of MDM2, p53, phosphorylated-MDM2, and phosphorylated-p53 in kidney of antiserum-induced GN mouse model and control animals was determined by Western blot analysis. $\beta$-Actin staining is shown as a loading control. The graph on the right shows the quantification of several Western blot analyses. B and C: MDM2 immunostaining (red) was performed on paraffin-embedded kidney samples of antiseruminduced GN mouse model and control adult C57BL/6 mice. B: Colocalization of MDM2 (arrows) with claudin (green) that stains the PECs. In antiseruminduced GN glomeruli MDM2 is strongly expressed in activated PECs (arrows). C: MDM2 (red) colocalization with CD31 stained glomerular endothelial cells (green). MDM2 expression is diminished in glomerular endothelial cells in antiserum-induced GN glomeruli. T0-PR0-3 stains nuclei in blue. ${ }^{*} P<0.05$, ${ }^{*} P<0.01$ versus baseline. Original magnification, $\times 630$. CTRL, control; GBM, glomerular basement membrane; GN, glomerulonephritis; MDM2, murine double minute-2; PEC, parietal epithelial cell. 


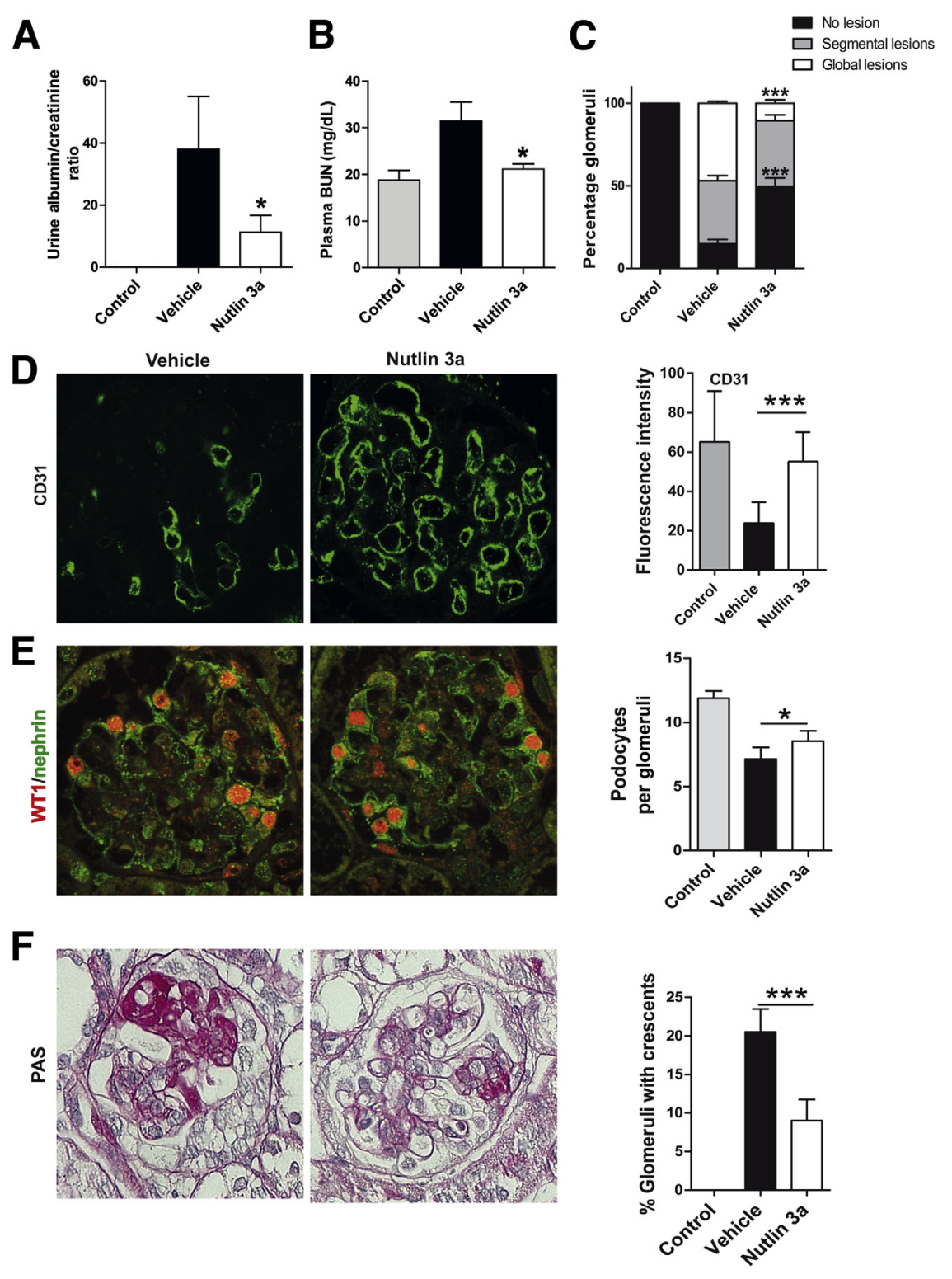

Figure 2 MDM2 blockade with the use of nutlin-3a ameliorates glomerular damage in antiserum-induced GN. A: Urine albumin/creatinine ratio. B: BUN concentrations were determined 7 days after sheep GBM antiserum injection. C: Glomerular injury was quantified on PAS-stained renal sections as described in Materials and Methods. D: Representative images of kidney sections stained for CD31 as a marker of glomerular capillaries and quantification of mean fluorescence area of CD31 positivity in glomeruli. E: Representative images of WT-1/nephrin costaining that were used to quantify differentiated podocytes in kidneys of vehicle- and nutlin-3a-treated animals. Numbers of nephrin/WT-1 double-positive cells were quantified in both groups at 7 days after induction of anti-GBM disease. F: Representative images of PAS staining showing amelioration of crescent formation and glomerulosclerosis in nutlin-3a-treated mice compared with control group and quantification of crescents from sections stained with PAS taken at day 7. Data are expressed as means $\pm \mathrm{SD}$. $n=5$ mice in each group. ${ }^{*} P<0.05,{ }^{* * *} P<0.005$, nutlin-3a- versus vehicle-treated mice. Original magnification: $\times 630$ $(\mathbf{D}$ and $\mathbf{E}) ; \times 400(\mathbf{F})$. BUN, blood urea nitrogen; GBM, glomerular basement membrane; GN, glomerulonephritis; MDM2, murine double minute-2, PAS, periodic acid-Schiff.

injection in C57BL/6 mice by Western blot analysis. Both MDM2 and p53 protein levels were unchanged in crescentic GN kidneys, whereas phosphorylated-MDM2 and phosphorylated-p53 were both significantly induced (Figure 1A and Supplemental Figure S1A). Immunostaining localized MDM2 mainly to the cytoplasm of PECs, GEnCs, and podocytes (Figure 1, B and C, and Supplemental Figure S1B). In antiserum-induced GN, glomerular capillary necrosis and podocyte loss reduced the MDM2 signal in endothelial cells and podocytes, but then strong MDM2 positivity occurred in proliferating PECs that formed crescents (Figure 1, B and C, and Supplemental Figure S1B). Together, MDM2 is expressed in GEnCs, podocytes, and PECs. Although total MDM2 protein remains the same, phosphorylated-MDM2 is significantly up-regulated in antiserum-induced GN kidney, suggesting the activation of MDM2 signaling.
Pre-emptive MDM2 Blockade with Nutlin-3a Suppresses Crescentic GN

Our model of crescentic GN is characterized by an increase in plasma creatinine concentration, BUN, albuminuria, and GEnC damage, leading to vascular necrosis and crescent formation and neutrophil and macrophage recruitment into the glomerulus within 7 days (Figure 2, A-F). To address a potential pathogenic role of MDM2 in this model we used nutlin-3a, a small molecule MDM2 inhibitor, that can block both its p53- and NF- $\kappa \mathrm{B}-$ dependent effects. ${ }^{7,8,11}$ Injection of $20 \mathrm{mg} / \mathrm{kg}$ nutlin-3a 24 hours before disease induction significantly reduced all of the aforementioned markers of crescentic GN (Figure 2, A-F). In particular, albuminuria, BUN, and glomerular lesions were significantly reduced by pre-emptive nutlin-3a treatment compared with vehicletreated mice (Figure 2, A-C). Moreover, nutlin-3a also 
A

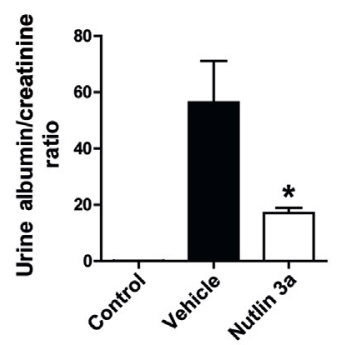

D

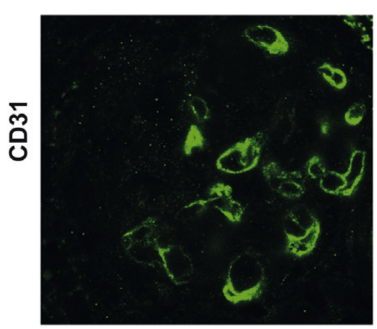

E

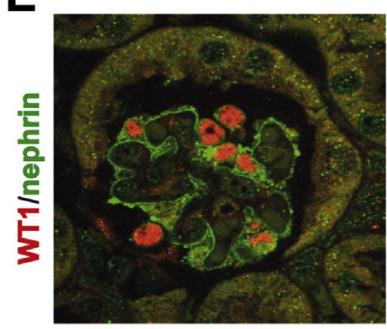

$\mathbf{F}$

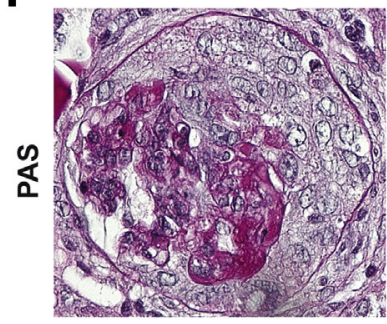

B

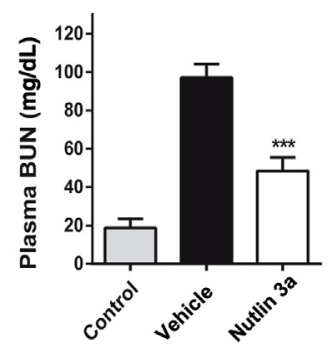

Nutlin
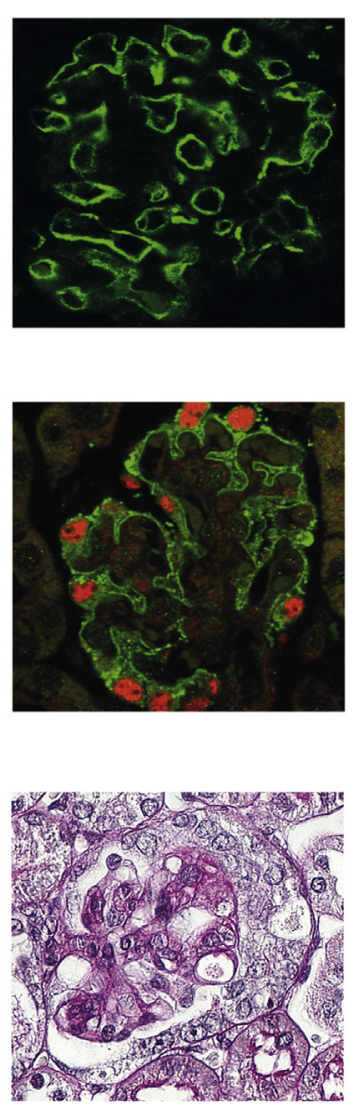
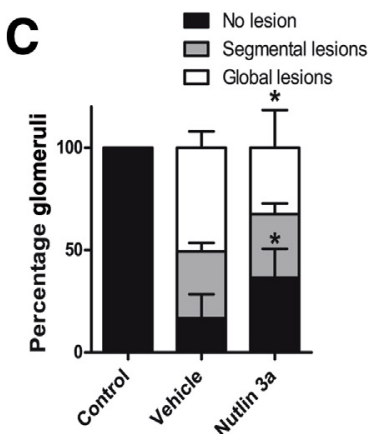

CD31
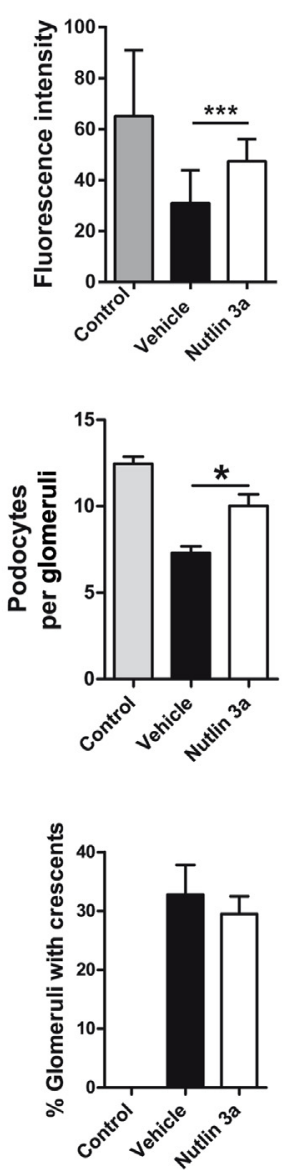

Figure 3 Nutlin-3a treatment suppresses most but not all of the pathologic features of crescentic $\mathrm{GN}$ in p53-deficient mice. A: Urine albumin/ creatinine ratio. B: BUN concentrations were determined 7 days after sheep GBM antiserum injection in $p 53$-deficient mice. C: Glomerular injury was quantified on PAS-stained renal sections as described in Materials and Methods. D: Representative images of kidney sections stained for CD31 as a marker of glomerular capillaries and a quantification of mean fluorescence area of CD31 positivity in glomeruli. E: Representative images of WT-1/nephrin costaining that was used to quantify differentiated podocytes in kidneys of vehicle- and nutlin-3a-treated kidneys. Numbers of nephrin/ WT-1 double-positive cells were quantified in both groups at 7 days after induction of anti-GBM disease. F: Representative images of PAS staining showing amelioration of crescent formation and glomerulosclerosis in nutlin-3a-treated mice compared with control group. Quantification of crescents from sections stained with PAS taken at day 7. Data are expressed as means \pm SD. $n=5$ mice in each group. ${ }^{*} P<0.05,{ }^{* *} P<0.005$, nutlin-3a- versus vehicle-treated mice. Original magnification: $\times 630(\mathbf{D}$ and $\mathbf{E}) ; \times 400(\mathbf{F})$. BUN, blood urea nitrogen; GBM, glomerular basement membrane; GN, glomerulonephritis; PAS, periodic acid-Schiff. largely prevented vascular lesions, as documented by endothelial CD31 positivity, the marker of glomerular vasculature, further prevented podocyte loss and crescent formation (Figure 2, D-F, and Supplemental Figure S2A). However, nutlin-3a treatment did not significantly affect glomerular thrombosis (Supplemental Figure S2C). Thus, preemptive MDM2 blockade with nutlin-3a suppresses GBM antiserum-induced crescentic GN.

\section{MDM2 Promotes Crescentic GN in a p53-Independent and p53-Dependent Manner}

Next we questioned whether the pathogenic effects of MDM2 in crescentic GN relate to its p53-dependent or -independent effects. To test this concept we performed the GBM antiserum-induced GN experiment in p53-deficient mice. The $p 53$-deficient mice did not display any kidney pathologic phenotype before the induction of the anti-GBM disease (Supplemental Figure S3). A comparison of the renal functional and histopathologic variables 7 days after GBM antiserum injection revealed that the pre-emptive nutlin-3a treatment had identical protective effects on GN-related plasma BUN, albuminuria, vascular necrosis, and podocyte loss (Figure 3, A-E, and Supplemental Figure S2B). Interestingly, only glomerular crescent formation was not affected by nutlin-3a treatment in p53-deficient mice (Figure 3F). These data imply that the pathogenic effects of MDM2 in crescentic GN are mostly p53-independent, with 
A

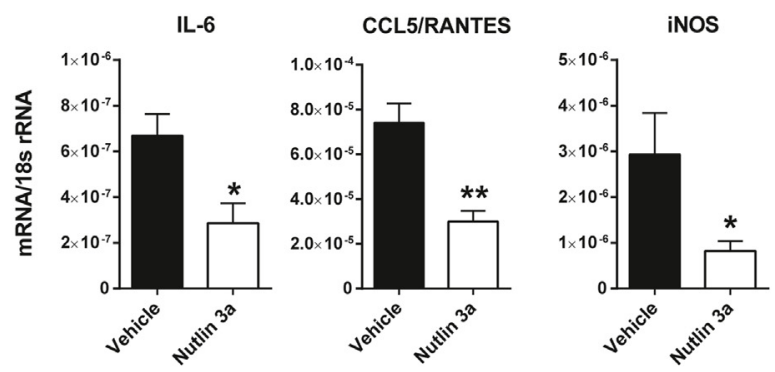

B
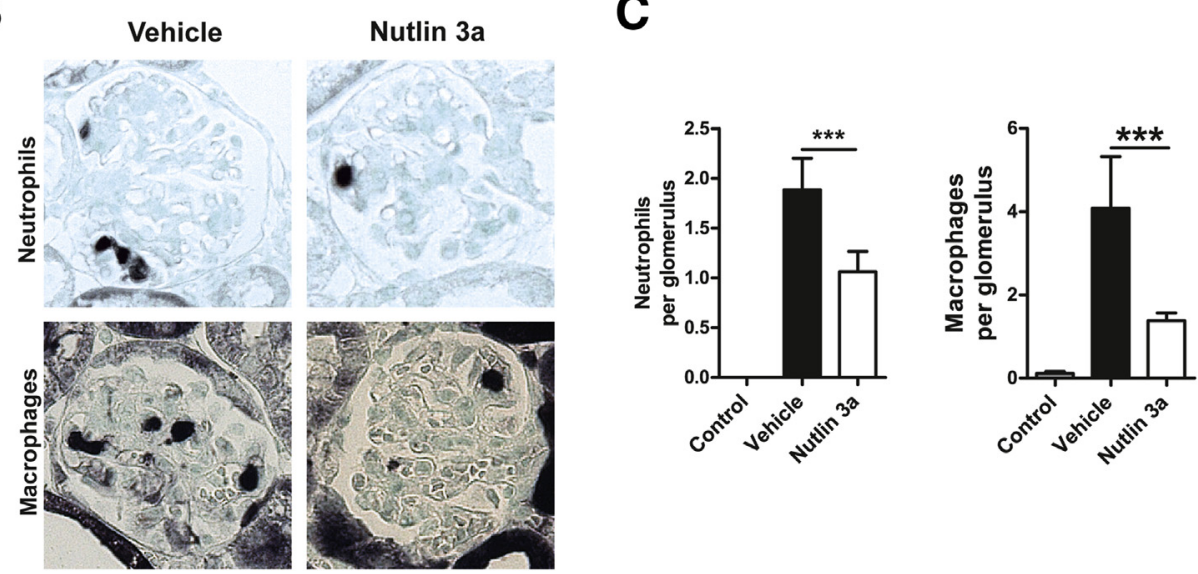

D

IL-6

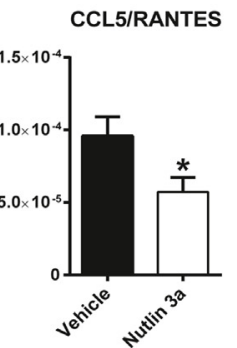

iNOS

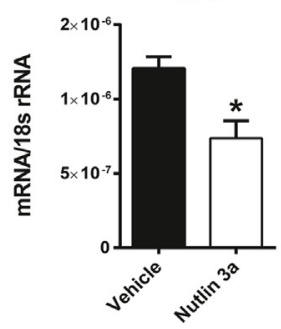

F

E

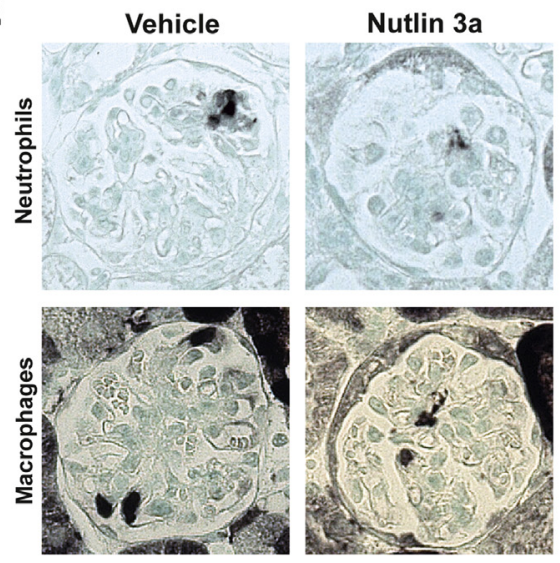

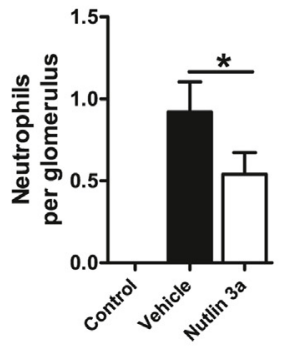

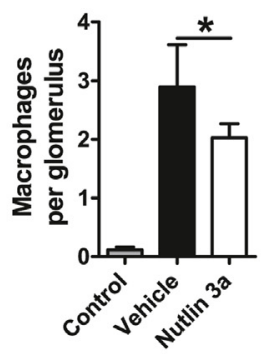

Figure 4 MDM2 blockade suppresses expression of inflammatory mediators and recruitment of leukocytes and macrophages in crescentic GN in wild-type and in p53-deficient mice. A-C: Antiserum-induced GN in wild-type C57BL/6 mice model. A: Total mRNA was prepared from vehicle- and nutlin-3a-treated kidneys. The mRNA levels were determined for the indicated cytokines and chemokines by real-time PCR and expressed as a mean of the ratio versus the respective 18s rRNA level. B: Representative images of kidney sections from vehicle- and nutlin-3a-treated kidneys stained for neutrophil and macrophages markers. C: Glomerular neutrophil (Ly-6B.2) and macrophage (mac-2) infiltrates are quantified by counting positive cells per glomerulus. D-F: Antiseruminduced GN in p53-deficient mice model. D: mRNA expression of inflammatory mediators in vehicle- and nutlin-3a-treated kidneys. E: Representative images of kidney sections from vehicle- and nutlin-3a-treated kidneys stained for neutrophil and macrophages markers. F: Glomerular neutrophil (Ly-6B.2) and macrophage (mac-2) infiltrates are quantified by counting positive cells per glomerulus. Data are expressed as means \pm SD. ${ }^{*} P<0.05,{ }^{*} P<0.01$, and ${ }^{* * *} P<0.005$ nutlin-3a versus vehicle group. Original magnification, $\times 400$ (B and E). CCL5, chemokine ligand 5; GN, glomerulonephritis; iNOS, inducible nitric oxide synthase; MDM2, murine double minute-2; RANTES, regulated on activation normal T cell expressed and secreted. 

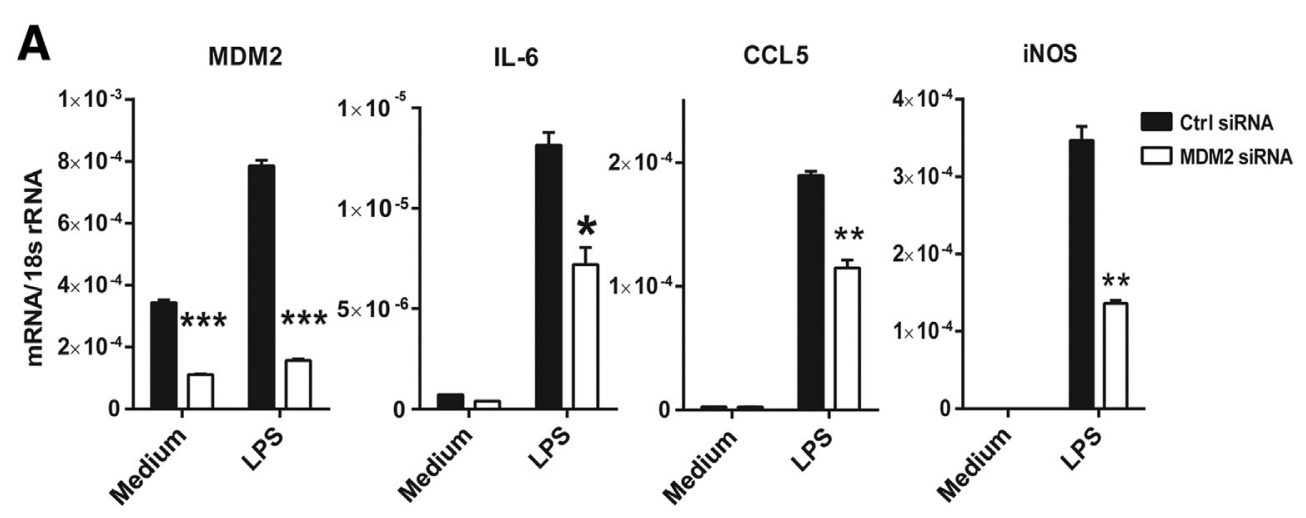

B

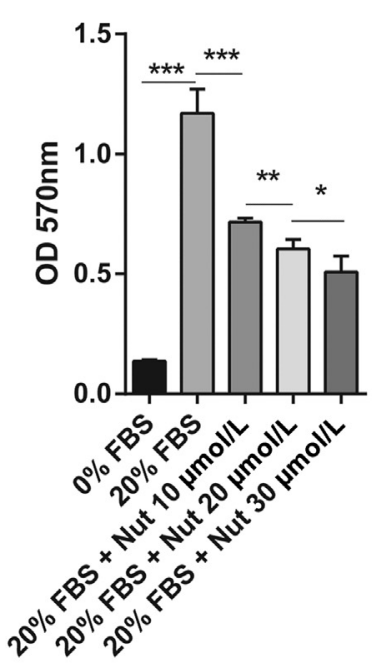

C

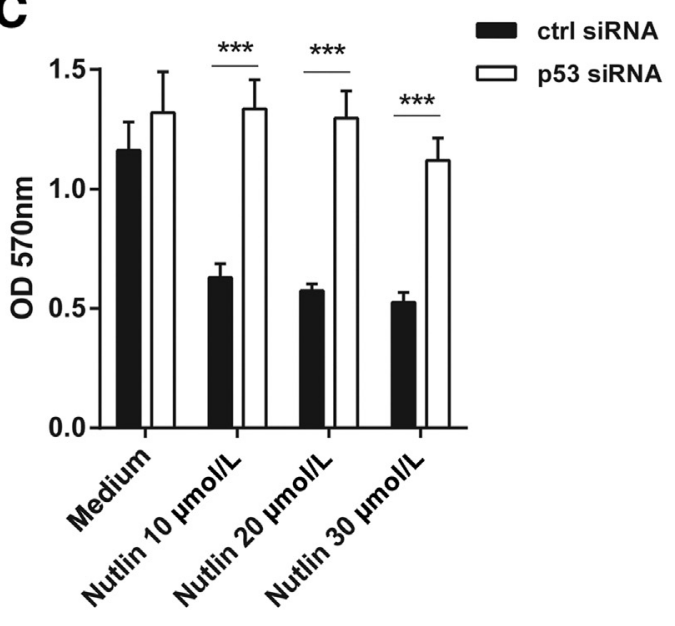

p53

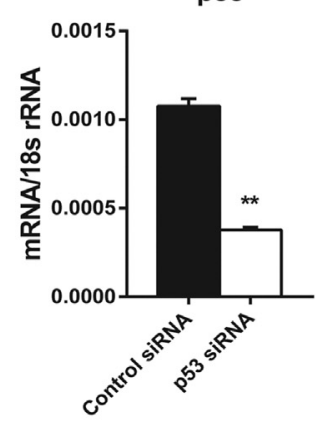

Figure 5 MDM2 inhibition in vitro suppresses inflammation in glomerular endothelial cells and proliferation of PECs. A: Total mRNA was prepared from GEnCs transfected with MDM2 siRNA and control siRNA and treated with LPS or vehicle. The mRNA levels were determined for the indicated cytokines and chemokines by real-time PCR and expressed as a mean of the ratio versus the respective $18 \mathrm{~s}$ rRNA level. B: PECs were grown in $0 \%$ or $20 \%$ serum-containing medium to confirm the proliferatory effect of serum on PECs. The proliferating PECs were then treated with increasing concentrations of MDM2 inhibitor Nut. Cell viability/ proliferation was determined after 24 hours by MTT assay. C: PECs were transfected with p53 siRNA and control siRNA and treated with increasing dose of Nut. The MTT assay shows complete reversal of Nut effect by p53 depletion in PECs. The graph below shows the efficiency of the p53 knockdown. All experiments were performed in triplicates. Data are expressed as means \pm SD. ${ }^{*} P<0.05,{ }^{*} P<0.01$, and ${ }^{* * *} P<0.005$. CCL5, chemokine ligand 5; Ctrl, control; FBS, fetal bovine serum; GEnC, glomerular endothelial cell; iNOS, inducible nitric oxide synthase; LPS, lipopolysaccharide; Nut, nutlin-3a; PEC, parietal epithelial cell.

the exception of crescent formation, which seems to involve a p53-dependent function of MDM2.

\section{MDM2 Promotes Renal Inflammation in a p53-Independent Manner}

p53-Independent effects of MDM2 may relate to its nonredundant role in NF- $\mathrm{KB}$ target gene expression. ${ }^{8}$ GBM antiserum-induced crescentic GN is a highly inflammatory disorder, involving the intrarenal expression of numerous NF- $\kappa \mathrm{B}$ target genes such as $I l 6, C c l 5$, or iNOS (Figure 4A). The up-regulation of these proinflammatory molecules was independent of the p53 genotype (Figure 4, A and D). Nutlin-3a treatment significantly reduced the intrarenal expression levels of these cytokines and chemokines at 7 days both in wild-type and in p53-deficient mice anti-GBM kidneys (Figure 4, A and D). To confirm the effect of

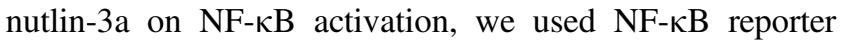

assay to monitor the NF- $\kappa \mathrm{B}$ signaling changes in GEnCs and PECs. We observed that nutlin-3a treatment suppressed the NF- $\kappa \mathrm{B}$ activation in both types of cells (Supplemental Figure S4). Further on we also observed similar effects in GEnCs where p53 was inhibited (Supplemental Figure S4), suggesting that nutlin-3a treatment suppressed the NF- $\kappa \mathrm{B}$ activation independent of $\mathrm{p} 53$. Because chemokines drive leukocyte recruitment that further contributes to local inflammation, we quantified glomerular neutrophils and macrophages by immunostaining. MDM2 blockade with nutlin-3a significantly reduced the infiltration of these proinflammatory cells into anti-GBM glomeruli in both groups of experimental animals (Figure 4, B, C, E, and F). It was reported previously that nutlin-3a promotes $\mathrm{p} 53$ activation and further down-regulates M2 gene expression. ${ }^{18}$ To address this, we performed real-time PCR analysis for the various M1 and M2 markers and CD163 and mannose receptor staining on the kidney sections. We found that 
A

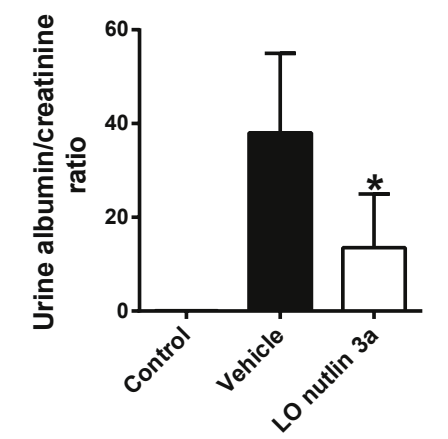

D

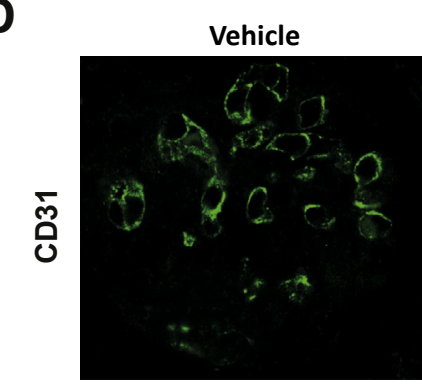

E

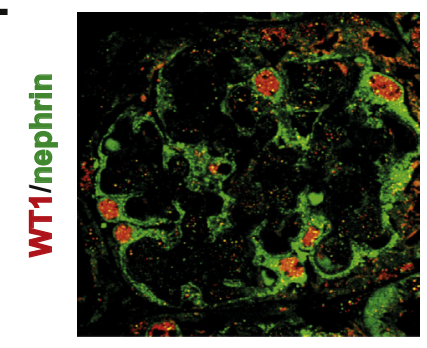

F

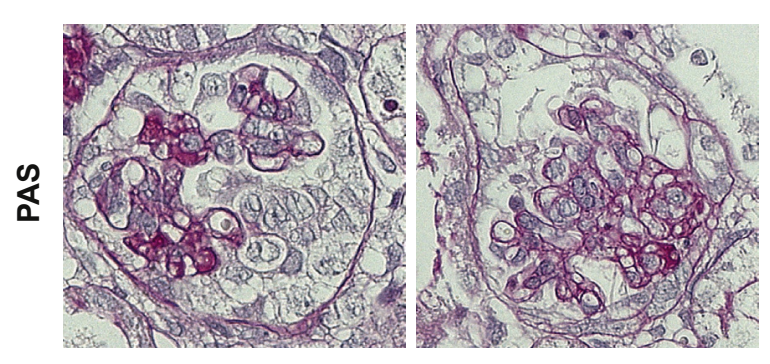

B

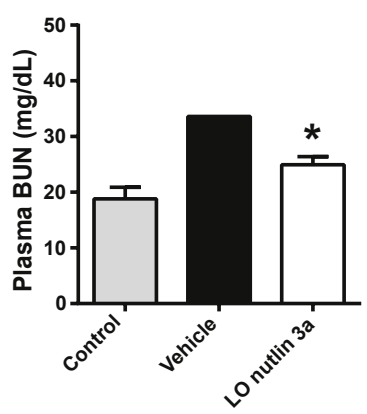

Nutlin
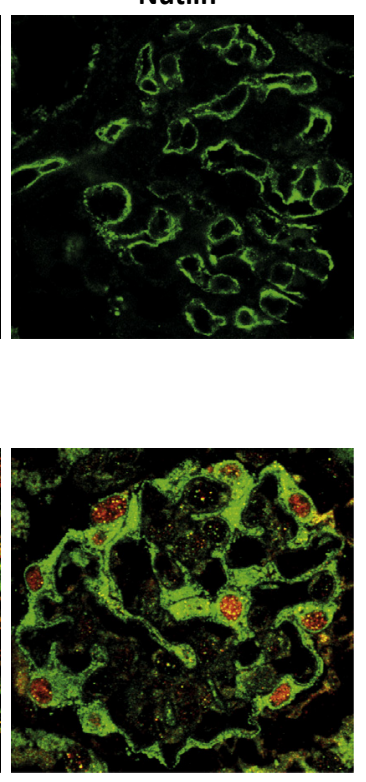

H

G

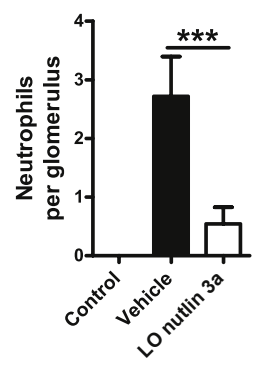

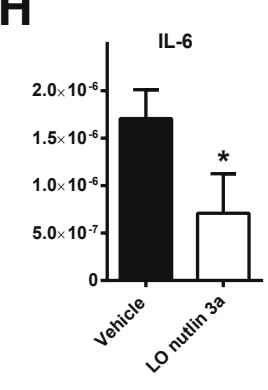

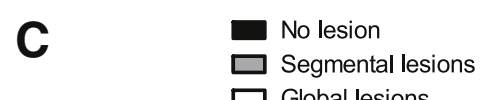

$\square$ Segmental lesions
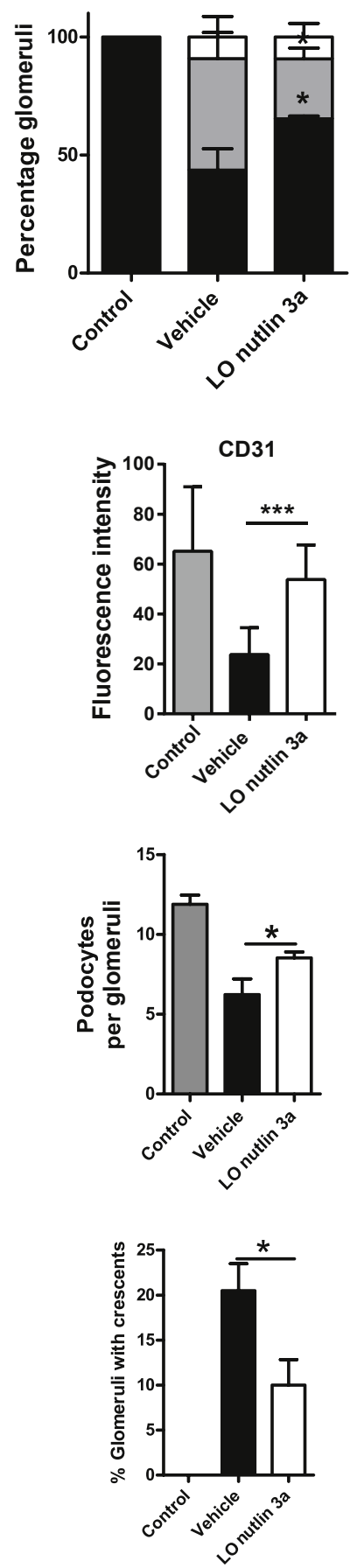

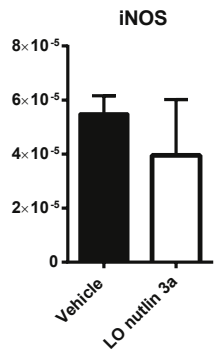


nutlin-3a treatment suppressed expression of both M1 and M2 markers in anti-GBM wild-type mice. In the $p 53-$ deficient mice model, we detected decline of several M1 markers on nutlin-3a treatment, but the M2 markers remained mostly unchanged. Furthermore, the CD163 and mannose receptor staining revealed a similar trend (Supplemental Figure S5, A-C, and Supplemental Figure S6, A-C). Together, MDM2 promotes renal inflammation in a $p 53$-independent manner.

\section{MDM2 Is Required for NF- $\kappa B$ Target Gene Transcription in GEnCs}

Crescentic GN involves a sterile inflammatory response with increased vascular permeability and expression of proinflammatory cytokines, chemokines, and adhesion molecules in GEnCs. ${ }^{19,20}$ Because we observed that MDM2 blockage attenuates glomerular endothelial injury and inflammation, we examined whether MDM2 modulates NF- $\kappa \mathrm{B}$ activation in GEnCs. We knocked down MDM2 expression with siRNA in murine GEnCs and measured mRNA expression levels of the NF- $\kappa \mathrm{B}$ target genes Il6, Ccl5, and iNOS. Because Toll-like receptor 4 (TLR4) stimulation by damageassociated molecular pattern molecules is known to be involved in pathogenesis of glomerular injury in crescentic GN, ${ }^{21}$ we used the TLR4 agonist LPS for GEnC stimulation to mimic the activation of this innate immune activation axis. Indeed, MDM2 depletion significantly suppressed the LPSinduced mRNA expression levels of IL-6, chemokine ligand 5, and inducible nitric oxide synthase in comparison with control siRNA-treated GEnCs (Figure 5A). Neither the unstimulated control GEnCs nor the unstimulated MDM2depleted GEnCs displayed up-regulation of the NF- $\kappa \mathrm{B}$ target genes (Figure $5 \mathrm{~A}$ ). Together, MDM2 promotes NF- $\kappa \mathrm{B}$ activation in the glomerular microvascular endothelium.

\section{MDM2 Mediates PEC Hyperplasia in a p53-Dependent Manner}

The formation of glomerular crescents is driven by hyperplasia of activated PECs, a process initiated by plasma components that leak from ruptured glomerular capillaries, ${ }^{22}$ by damageassociated molecular pattern molecules released from dying glomerular cells, ${ }^{3,23}$ and by local growth factor secretion. ${ }^{20}$ Because MDM2 is highly expressed in PECs and PEC hyperplasia was susceptible to nutlin-3a in wild-type but not in p53-deficient mice, we speculated that MDM2 has a p53dependent role in PEC hyperplasia. To test this concept we cultured murine PECs in serum-free medium and in medium that contained $20 \%$ serum, which confirmed the mitogenic effect of serum on PECs (Figure 5B). Further on, treatment with nutlin-3a dose dependently suppressed serum-induced PEC proliferation (Figure 5B). To test whether this MDM2 effect was p53-dependent we used siRNA to suppress p53 transcripts in PECs before treating them with increasing doses of nutlin-3a. Although control siRNA-treated PECs had no effect on nutlin-3a-mediated inhibition of PEC proliferation, this effect was completely abrogated with $\mathrm{p} 53$-specific siRNA (Figure 5C). Thus, MDM2 promotes serum-induced hyperplasia of PECs in a p53-dependent manner.

\section{Delayed Onset of Nutlin-3a Treatment Is Still Protective in Severe GN}

The aforementioned data document synergistic pathogenic effects of p53-dependent and -independent biological functions of MDM2 in crescentic GN, implying that MDM2 could be a potential therapeutic target in crescentic GN. Validating therapeutic targets in crescentic GN requires an onset of the therapeutic intervention not before the disease is established, because indicated by clinically relevant biomarkers of GN such as proteinuria and potentially also impaired renal excretory function. ${ }^{24}$ To test whether nutlin-3a is also capable of suppressing established GN we initiated nutlin-3a treatment 48 hours after antiserum injection, a time point when massive proteinuria and elevated BUN were already established (Supplemental Figure S7). Nutlin-3a treatment significantly reduced albuminuria, BUN, and glomerular injury at day 7 (Figure 6, A-C). It also significantly ameliorated the extent of glomerular capillary necrosis, podocyte loss, and crescent formation (Figure 6, D-F, and Supplemental Figure S8). The glomerular recruitment of neutrophils and macrophages and the induction of proinflammatory genes were also significantly reduced (Figure 6, G and $\mathrm{H}$ ). Together, these data document that even a delayed onset of MDM2 blockade with nutlin-3a can improve the outcome of established GN.

\section{Discussion}

We hypothesized that MDM2 drives crescentic GN by $\mathrm{NF}-\kappa \mathrm{B}-$ dependent glomerular inflammation and by

Figure 6 Late onset of MDM2 blockade by nutlin-3a still ameliorates antiserum-induced GN. A: Urine albumin/creatinine ratio. B: BUN concentrations were determined 7 days after sheep GBM antiserum injection. Nutlin-3a injections were initiated 48 hours after induction of antiserum GN. C: Glomerular injury was quantified on PAS-stained renal sections as described in Materials and Methods. D: Representative images of kidney sections stained for CD31 as a marker of glomerular capillaries and quantification of mean fluorescence area of CD31 positivity in glomeruli. E: Representative images of WT-1/nephrin costaining that were used to quantify differentiated podocytes in kidneys of vehicle- and nutlin-3a-treated animals. Numbers of nephrin/WT-1 double-positive cells were quantified in both groups at 7 days after induction of anti-GBM disease. F: Representative images of PAS staining showing amelioration of crescent formation and sclerotization of glomeruli in nutlin-3a-treated mice compared with control group. Quantification of crescents from sections stained with PAS taken at day 7. G: Glomerular neutrophil (Ly-6B.2) and macrophage (mac-2) infiltrates are quantified by counting of positive cells per glomerulus. H: The mRNA expression of inflammatory mediators in vehicle- and nutlin-3a-treated kidneys. Data are expressed as means \pm SD. $n=5$ mice in each group. ${ }^{*} P<0.05,{ }^{* *} P<0.005$, nutlin-3a- versus vehicle-treated mice. Original magnification: $\times 630(\mathbf{D}$ and $\mathbf{E}) ; \times 400(\mathbf{F})$. BUN, blood urea nitrogen; CCL5, chemokine ligand 5; GBM, glomerular basement membrane; GN, glomerulonephritis; iNOS, inducible nitric oxide synthase; L0, late onset; MDM2, murine double minute-2; PAS, periodic acid-Schiff. 
p53-dependent PEC hyperproliferation. Our data confirmed this concept and found that MDM2 blockade ameliorated crescentic GN even when MDM2 inhibition is started after the onset of GN. Thus, MDM2 blockade represents a novel potential therapeutic strategy in severe crescentic GN.

We have previously identified a dual role of MDM2, that is, a proinflammatory effect by modulating NF- $\kappa \mathrm{B}$ signaling and a pro-proliferative effect by blocking p53-mediated cell death in the postischemic kidney. ${ }^{7}$ Because crescentic GN, regardless of its cause, is characterized by massive sterile inflammation, we hypothesized that MDM2 blockade with nutlin-3a might protect from inflammation-related glomerular damage. Indeed, our data indicate that MDM2 inhibition blocks the induction of proinflammatory cytokines and chemokines and neutrophil and macrophage recruitment in the glomerulus during crescentic GN. This is consistent with previous reports indicating that MDM2 blockade with nutlin-3a suppresses LPS-induced lung inflammation ${ }^{25}$ or ameliorates human atherosclerosis by suppression of NF$\kappa \mathrm{B}$-dependent inflammation in vascular smooth muscle cells. $^{26,27}$ In the kidney, MDM2 inhibition prevents glomerular inflammation and podocyte loss in early Adriamycin nephropathy ${ }^{12}$ and postischemic induction of proinflammatory cytokines and chemokines and the subsequent recruitment of leukocytes to the site of injury in renal ischemia-reperfusion injury model. ${ }^{7}$ The sterile inflammatory response associates with increased vascular permeability, up-regulation of proinflammatory molecules in GEnCs and subsequent glomerular capillary necrosis. ${ }^{19,20}$ Our in vivo data indicate that MDM2 inhibition prevents GEnC damage and necrosis in glomerular microvasculature in GBM antiserum-induced crescentic GN. Moreover, we show that MDM2 inhibition suppresses TLR4 agonistmediated induction of proinflammatory molecules in cultured murine GEnCs. This implies the involvement of MDM2 in promoting inflammation in GEnCs by the TLR4 axis. The TLR4 activation by damage-associated molecular pattern molecules is known to be involved in pathogenesis of glomerular injury in crescentic GN. ${ }^{21}$ Furthermore, MDM2 blockade also limits loss of podocytes, proteinuria, and glomerulosclerosis in our model. We can speculate that the highly inflammatory milieu in the GBM antiserum-induced crescentic GN contributes to the podocyte damage. It is known that the damaged postmitotic podocytes in many renal diseases can be driven into mitosis that cannot be successfully completed and thus results in podocyte cell death by mitotic catastrophe. ${ }^{28}$ Our data indicate that suppression of p53-dependent or NF- $\kappa \mathrm{B}$-dependent MDM2 function prevents podocyte demise. We can speculate that at least part of the podocyte rescue in crescentic GN model, similarly to the Adriamycin nephropathy model, occurs because of MDM2 blockadedriven cell cycle arrest in podocytes through an increase of p53 and p21 protein levels to prevent mitotic catastrophe. ${ }^{12}$ Furthermore, inhibition of NF- $\kappa \mathrm{B}$ overactivation by nutlin-3a also contributes to survival of podocytes because we observed diminished podocyte death in $p 53$-deficient mouse model as well. ${ }^{7}$ As such, nutlin-3a suppresses podocyte injury such as other NF- $\kappa \mathrm{B}$ antagonists through reduced monocyte/ macrophage infiltration and ameliorated inflammation. ${ }^{29-32}$

Our data indicate that the pathogenic effects of MDM2 in crescentic GN are largely p53 independent because most of the end point variables were ameliorated by nutlin-3a blockade of MDM2 also in p53-deficient mice. Because we observed the amelioration of the glomerular pathologic phenotype in the nutlin-3a-treated p53-deficient mice, one would expect that the PECs receive less pro-proliferative signals; therefore, fewer crescents are formed. ${ }^{22}$ Interestingly, serum-induced PEC proliferation and cellular crescents were significantly reduced by nutlin-3a treatment in wild-type but not in p53-deficient mice. This implies that nutlin-3a blocks serum-induced PEC proliferation in a p53-dependent manner. The observation that nutlin-3a did not affect PEC proliferation (crescent formation) in p53deficient mice is consistent with our previous study on the reactive proliferative response of surviving tubular epithelial cells of p53-deficient mice recovering from acute tubular necrosis. ${ }^{7}$ We assume that the lack of p53 leads to accelerated epithelial proliferation so that a lower number of crescents that result from attenuated concomitant glomerular tuft injury is partially compensated by PEC hyperproliferation so that the number of crescents is maintained despite glomerular injury. However, the exact mechanism for this phenomenon warrants further thorough investigation. In addition, our in vitro experiments confirm that serum-induced proliferation of PECs can be suppressed by nutlin-3a in a p53-dependent manner, because p53 suppression abrogates antiproliferative effect of nutlin-3a. This is consistent with those studies that reported the necessity of functional p53 for the growth inhibition of oncogenic cells driven by MDM2. ${ }^{9}$ Mutation or depletion of p53 results in pathologic overgrowth of cancer cells with uncontrolled MDM2 expression, which can no longer be blocked with nutlin-3a. ${ }^{33}$ Similarly, MDM2 inhibition with nutlin-3a attenuates neointimal hyperplasia after vascular injury in mice with intact $\mathrm{p} 53^{26}$ or abrogates the abnormal proliferation of lymphocytes in systemic lupus erythematosus in a p53dependent manner. ${ }^{13}$

Our proof-of-concept experiments were based on preemptive MDM2 inhibition with nutlin-3a. To explore a potential efficacy of nutlin treatment in severe GN, we also applied nutlin-3a treatment after GN induction. Delayed onset of nutlin-3a treatment was equally protective as preemptive therapy in terms of glomerular injury, proteinuria, BUN concentrations, and inflammation.

Together, MDM2 drives severe GN by NF- $\kappa \mathrm{B}-$ dependent glomerular inflammation, which accelerates glomerular vascular necrosis and podocyte loss and by $\mathrm{p} 53$-dependent PEC proliferation with crescent formation. Both pre-emptive and delayed onset of MDM2 blockade with nutlin-3a abrogates all aspects of GBM antiserum-induced severe GN. Therefore, we conclude that MDM2 blockade could represent a novel therapeutic strategy in severe GN. 


\section{Supplemental Data}

Supplemental material for this article can be found at http://dx.doi.org/10.1016/j.ajpath.2016.01.017.

\section{References}

1. Greenhall GH, Salama AD: What is new in the management of rapidly progressive glomerulonephritis? Clin Kidney J 2015, 8:143-150

2. Henique C, Papista C, Guyonnet L, Lenoir O, Tharaux PL: Update on crescentic glomerulonephritis. Semin Immunopathol 2014, 36: 479-490

3. Kumar SV, Kulkarni OP, Mulay SR, Darisipudi MN, Romoli S, Thomasova D, Scherbaum CR, Hohenstein B, Hugo C, Muller S, Liapis H, Anders HJ: Neutrophil extracellular trap-related extracellular histones cause vascular necrosis in severe GN. J Am Soc Nephrol 2015, 26:2399-2413

4. Couser WG: Basic and translational concepts of immune-mediated glomerular diseases. J Am Soc Nephrol 2012, 23:381-399

5. Moeller MJ, Smeets B: Role of parietal epithelial cells in kidney injury: the case of rapidly progressing glomerulonephritis and focal and segmental glomerulosclerosis. Nephron Exp Nephrol 2014, 126:97

6. Linkermann A, Stockwell BR, Krautwald S, Anders HJ: Regulated cell death and inflammation: an auto-amplification loop causes organ failure. Nat Rev Immunol 2014, 14:759-767

7. Mulay SR, Thomasova D, Ryu M, Anders HJ: MDM2 (murine double minute-2) links inflammation and tubular cell healing during acute kidney injury in mice. Kidney Int 2012, 81:1199-1211

8. Thomasova D, Mulay SR, Bruns H, Anders HJ: p53-independent roles of MDM2 in NF-kappaB signaling: implications for cancer therapy, wound healing, and autoimmune diseases. Neoplasia 2012, 14:1097-1101

9. Vazquez A, Bond EE, Levine AJ, Bond GL: The genetics of the p53 pathway, apoptosis and cancer therapy. Nat Rev Drug Discov 2008, 7 : 979-987

10. Leach FS, Tokino T, Meltzer P, Burrell M, Oliner JD, Smith S, Hill DE, Sidransky D, Kinzler KW, Vogelstein B: p53 Mutation and MDM2 amplification in human soft tissue sarcomas. Cancer Res 1993, $53: 2231-2234$

11. Vassilev LT, Vu BT, Graves B, Carvajal D, Podlaski F, Filipovic Z, Kong N, Kammlott U, Lukacs C, Klein C, Fotouhi N, Liu EA: In vivo activation of the p53 pathway by small-molecule antagonists of MDM2. Science 2004, 303:844-848

12. Mulay SR, Thomasova D, Ryu M, Kulkarni OP, Migliorini A, Bruns H, Grobmayr R, Lazzeri E, Lasagni L, Liapis H, Romagnani P, Anders HJ: Podocyte loss involves MDM2-driven mitotic catastrophe. J Pathol 2013, 230:322-335

13. Allam R, Sayyed SG, Kulkarni OP, Lichtnekert J, Anders HJ: Mdm2 promotes systemic lupus erythematosus and lupus nephritis. J Am Soc Nephrol 2011, 22:2016-2027

14. Suresh K: An overview of randomization techniques: an unbiased assessment of outcome in clinical research. J Hum Reprod Sci 2011, 4: $8-11$

15. European Parliament, Council of the European Union: Directive 2010/63/EU of the European Parliament and of the council of 22 September 2010 on the protection of animals used for scientific purposes. Available at http://eur-lex.europa.eu/LexUriServ/LexUriServ.do? uri=OJ:L:2010:276:0033:0079:eN:PDF (accessed September 2015)

16. Ohse T, Pippin JW, Vaughan MR, Brinkkoetter PT, Krofft RD, Shankland SJ: Establishment of conditionally immortalized mouse glomerular parietal epithelial cells in culture. J Am Soc Nephrol 2008, 19:1879-1890
17. Akis N, Madaio MP: Isolation, culture, and characterization of endothelial cells from mouse glomeruli. Kidney Int 2004, 65: 2223-2227

18. Li L, Ng DS, Mah WC, Almeida FF, Rahmat SA, Rao VK, Leow SC, Laudisi F, Peh MT, Goh AM, Lim JS, Wright GD, Mortellaro A, Taneja R, Ginhoux F, Lee CG, Moore PK, Lane DP: A unique role for p53 in the regulation of M2 macrophage polarization. Cell Death Differ 2015, 22:1081-1093

19. Atkins RC, Nikolic-Paterson DJ, Song Q, Lan HY: Modulators of crescentic glomerulonephritis. J Am Soc Nephrol 1996, 7:2271-2278

20. Bollee G, Flamant M, Schordan S, Fligny C, Rumpel E, Milon M, Schordan E, Sabaa N, Vandermeersch S, Galaup A, Rodenas A, Casal I, Sunnarborg SW, Salant DJ, Kopp JB, Threadgill DW, Quaggin SE, Dussaule JC, Germain S, Mesnard L, Endlich K, Boucheix C, Belenfant X, Callard P, Endlich N, Tharaux PL: Epidermal growth factor receptor promotes glomerular injury and renal failure in rapidly progressive crescentic glomerulonephritis. Nat Med 2011, 17:1242-1250

21. Giorgini A, Brown HJ, Sacks SH, Robson MG: Toll-like receptor 4 stimulation triggers crescentic glomerulonephritis by multiple mechanisms including a direct effect on renal cells. Am J Pathol 2010, 177: 644-653

22. Ryu M, Migliorini A, Miosge N, Gross O, Shankland S, Brinkkoetter PT, Hagmann H, Romagnani P, Liapis H, Anders HJ: Plasma leakage through glomerular basement membrane ruptures triggers the proliferation of parietal epithelial cells and crescent formation in noninflammatory glomerular injury. J Pathol 2012, 228:482-494

23. Sicking EM, Fuss A, Uhlig S, Jirak P, Dijkman H, Wetzels J, Engel DR, Urzynicok T, Heidenreich S, Kriz W, Kurts C, Ostendorf T, Floege J, Smeets B, Moeller MJ: Subtotal ablation of parietal epithelial cells induces crescent formation. J Am Soc Nephrol 2012, 23:629-640

24. Anders HJ, Vielhauer V: Identifying and validating novel targets with in vivo disease models: guidelines for study design. Drug Discov Today 2007, 12:446-451

25. Liu G, Park YJ, Tsuruta Y, Lorne E, Abraham E: p53 Attenuates lipopolysaccharide-induced NF-kappaB activation and acute lung injury. J Immunol 2009, 182:5063-5071

26. Hashimoto $T$, Ichiki $T$, Ikeda $J$, Narabayashi E, Matsuura $H$, Miyazaki R, Inanaga K, Takeda K, Sunagawa K: Inhibition of MDM2 attenuates neointimal hyperplasia via suppression of vascular proliferation and inflammation. Cardiovasc Res 2011, 91:711-719

27. Ihling C, Haendeler J, Menzel G, Hess RD, Fraedrich G, Schaefer HE, Zeiher AM: Co-expression of p53 and MDM2 in human atherosclerosis: implications for the regulation of cellularity of atherosclerotic lesions. J Pathol 1998, 185:303-312

28. Lasagni L, Lazzeri E, Shankland SJ, Anders HJ, Romagnani P: Podocyte mitosis - a catastrophe. Curr Mol Med 2013, 13:13-23

29. Muller DN, Dechend R, Mervaala EM, Park JK, Schmidt F, Fiebeler A, Theuer J, Breu V, Ganten D, Haller H, Luft FC: NF-kappaB inhibition ameliorates angiotensin II-induced inflammatory damage in rats. Hypertension 2000, 35:193-201

30. Rangan GK, Wang Y, Tay YC, Harris DC: Inhibition of nuclear factorkappaB activation reduces cortical tubulointerstitial injury in proteinuric rats. Kidney Int 1999, 56:118-134

31. Rangan GK, Wang Y, Tay YC, Harris DC: Early administration of PDTC in adriamycin nephropathy: effect on proteinuria, cortical tubulointerstitial injury, and NF-kappaB activation. Ren Fail 2001, 23: 773-780

32. Tamada S, Nakatani T, Asai T, Tashiro K, Komiya T, Sumi T, Okamura M, Kim S, Iwao H, Kishimoto T, Yamanaka S, Miura K: Inhibition of nuclear factor-kappaB activation by pyrrolidine dithiocarbamate prevents chronic FK506 nephropathy. Kidney Int 2003, 63:306-314

33. Duffy MJ, Synnott NC, McGowan PM, Crown J, O'Connor D, Gallagher WM: p53 as a target for the treatment of cancer. Cancer Treat Rev 2014, 40:1153-1160 